Ann. Zootech., I968, 17 (3), 23I-244.

I.N.R.A.
BIBLIOTHEQUE UO :?
DOMAINE OE CROUE.
G3039
CLERMONT-FD CEOEX

\title{
EFFETS DE LA SUPPRESSION DE LA TRAITE DU DIMANCHE SOIR, CHEZ LES BOVINS DE RACE FRANÇAISE FRISONNE PIE NOIRE
}

\author{
J. LABUSSIERE, J. COINDET \\ avec la collaboration technique de J.F. Combaud \\ F.-A. de la Chevalerie et J. Pont \\ Laboratoire de Physiologie de la Lactation, \\ Centre national de Recherches zootechniques, 78--Jouy-en-Josas \\ Institut national de la Recherche agronomique \\ Lycée agricole, $\gamma 6$ - Yvetot
}

\section{SOMMAIRE}

La suppression de la traite du dimanche soir a été effectuée pendant toute une lactation chez I 4 vaches Française Frisonne Pie Noire. Elle n'a pas été accompagnée de modifications de l'horaire des autres traites.

Comparées à celles du lot témoin, les productions en 300 jours des lipides, des matières azotées, du lactose, de l'extrait sec et du lait, sont respectivement réduites de 6,3 P. I००, 6,6 p. 1००, 7,8 p. I००, 7,1 p. roo et 7,5 p. Ioo.

La sécrétion étant fortement perturbée (en quantité et en qualité) pendant les 48 heures qui suivent cette omission, le passage du contrôleur laitier avant le mardi soir est donc exclı, dans les fermes où cette technique serait adoptée. Nous n'avons pas constaté, même à long terme, de modifications de l'état sanitaire de la mamelle.

\section{I. - INTRODUCTION}

Le taux horaire de sécrétion du lait semble pratiquement inchangé tant que l'écart séparant deux traites n'excède pas I6 heures (EI,IOT, I959), la production quotidienne restant effectivement la même, que l'on choisisse des espacements égaux (I2 h-I2 h) ou inégaux ( $8 \mathrm{~h}$-I $6 \mathrm{~h}$ ), (voir revue bibliographique de LABUSSIÈRE et RICHARD, I965).

L'accumulation du lait dans le pis pendant une période plus longue, se traduit par une baisse de l'activité des cellules mammaires, et au bout de 24 heures, la quantité de lait recueillie est inférieure d'environ Io à I2 p. Ioo à celle que l'on aurait pu espérer si la linéarité mentionnée ci-dessus avait été maintenue. 
Io Ibit-on penser pour autant que la perte constatée lors de la pratique journalière d'une seule traite, est aussi faible que cela ?

Les résultats prouvent qu'il n'en est rien puisqu'en répétant une telle méthode pendant plusieurs mois, WOODWARI) (I93I) aboutit à des chiffres beaucoup plus élevés et moins encourageants (50 p. IOO).

Cette perte varie d'ailleurs énormément:

- suivant les vaches, de 25 à 89 p. Ioo pour ClaAssson et al. (I959);

- suivant leur stade physiologique, la production paraissant moins affectée vers la fin de la lactation (HESSELTINE et al., I953; CLAESSON et al., I959).

La traite une seule fois par jour, un ou deux mois avant le tarissement, offre relativement peu d'intérêt, l'agriculteur étant toujours astreint à une présence constante par les vaches qui n'ont pas encore atteint l'époque favorable à l'application de cette technique.

Ainsi, la traite quoticlienne unique pendant toute la lactation ne peut être économiquement justifiée tant qu'une étude n'aura pas permis de distinguer et d'expliquer les différences individuelles pour orienter la sélection.

$2^{\circ}$ En revanche, l'introduction une fois par semaine d'un intervalle de 24 heures, offre probablement moins de risques. Ceci permettrait réellement de libérer momentanément l'éleveur ; c'est la raison pour laquelle la suppression de la traite du dimanche soir nous paraît finalement être pour l'agriculteur, et dans l'immédiat, la technique la plus intéressante.

Il y a deux manières d'envisager cette suppression :

a) La première est conçue de façon à ménager un intervalle de 8 heures entre la traite du samedi soir et celle du dimanche matin, et également de i 8 heures entre cette traite tardive du dimanche et celle plus matinale du lundi. C'est cette solution qui a été expérimentée en Allemagne parallèlement par Wr'TT (I963) au cours de deux essais de $\delta$ et $I_{5}$ semaines, et par BoGNFR ( g $^{6} 3$ ) sur une période encore plus courte.

Les résultats qui sont présentés dans les deux essais sont toutefois difficiles à interpréter, car ils ne portent que sur des laps de temps très réduits ( 4 \% h ou une semaine), et ne tiennent pas compte ainsi de l'effet cumulatif de rétentions 1sctées répétées.

b) La seconde est celle que nous avons adoptée car elle présentait plus de risques tant en ce qui concerne la production laitière, que l'état sanitaire de la mamelle. Elle consiste à supprimer carrément la traite du dimanche soir, laissant ainsi l'animal sans traite pendant 24 heures consécutives puisque les horaires habituellement observés les autres matins n'ont pas été modifiés.

C'est ce schéma qui a été utilisé par At'rREY, RoLImss et CANnon (Ig63) lors d'une expérience malheureusement trop brève et portant sur un nombre trop faible d'animaux.

CrAEssox (I965) a procédé de la même façon de la parturition jusqu'au tarissement chez les génisses, mais uniquement à partir de la $6^{\mathbf{e}}$ semaine de lactation chez les vaches plus âgées.

Au cours de l'expérience qui va être exposée maintenant, toutes les vaches, quel que soit leur âge ou leur niveau de production laitière, n'ont pas été traites le dimanche soir, dès le vêlage et pendant toute la lactation. 


\section{II. - MATÉRIEI, ET MÉTHODES}

\section{A. - Animaux}

\section{La première année (1964-1965).}

Quatorze vaches Françaises Frisonne Pie Noire (tab1. I) furent réparties le plus équitablement possible en deux lots, compte tenu :

- du numéro de lactation,

- de la date de mise bas, génisses).

- des productions laitières précédentes (dans le cas où il ne s'agissait pas de

- I,e lot témoin a toujours été trait deux fois par jour à 6 heures et I 6 h 30 .

- Le lot expérimental a été soumis aux mêmes horaires à l'exception du dimanche soir où la traite n'a pas été effectuée.

TABI,EAU I

Réparition des animaux

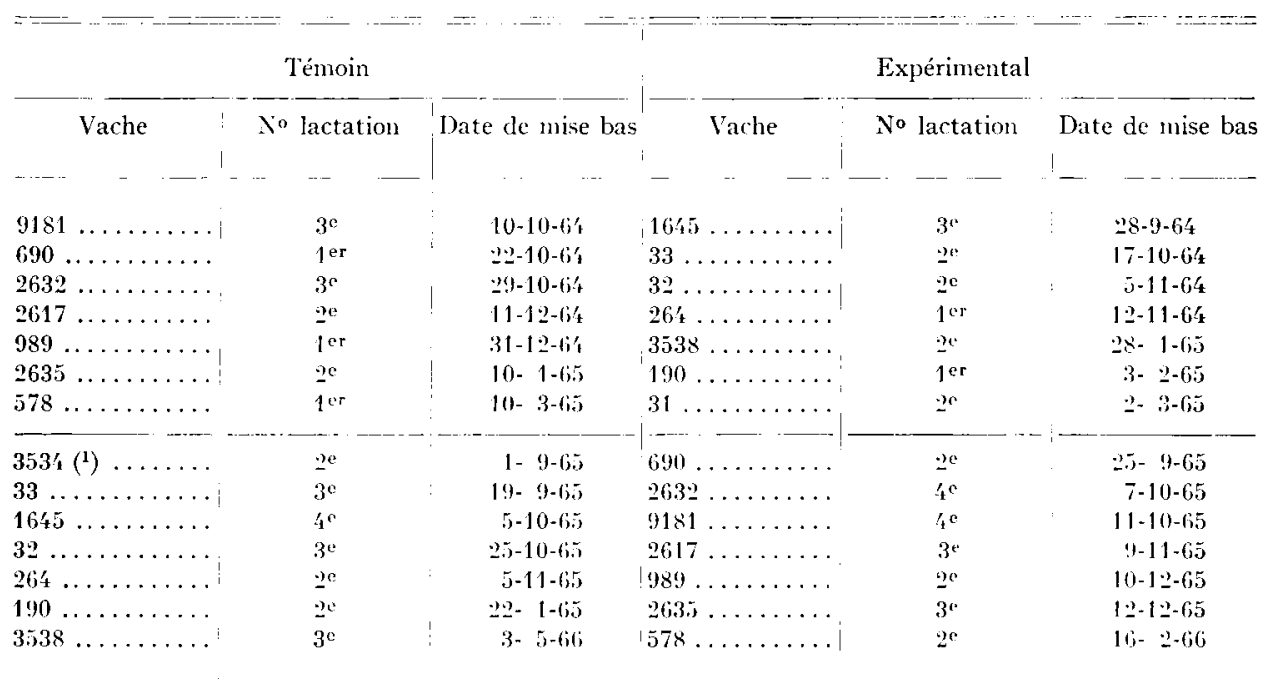

(1) La vache 3534 a remplacé la vache 31 qui n'était pas gestante la deuxième année de lexpérience. Ces 2 animaux avaient une production sensiblement équivalente en lere lactation (respectivement $\geq 836 \mathrm{et} 2709 \mathrm{~kg}$ en $300 \mathrm{j}$ ).

\section{La seconde année (1965-1966).}

Au fur et à mesure de lactations nouvelles, le lot expérimental est devenu témoin et inversement le lot témoin est devenu expérimental (tabl. I).

En régime hivernal, il fut distribué à tous les animaux une ration de base constituée de $12 \mathrm{~kg}$ de foin de pré et $\mathrm{r} 5 \mathrm{~kg}$ d'ensilage de prairie naturelle.

Afin que chaque vache puisse exprimer pleinement ses potentialités, le complé- 
ment a été apporté en concentré et calculé sur la base d'une production de $2 \mathrm{~kg}$ supérieure à la production réelle.

\section{B. - Technique de traite}

La traite fut effectuée avec une machine Alfa-I_aval $(33 \mathrm{~cm} \mathrm{Hg}$, rapport I/I, 45 pulsations/minute). Un massage de la mamelle avec un linge préalablement trempé dans de l'eau à $50^{\circ}$ précédait la pose des gobelets d'environ une minute.

Les dernières fractions de lait furent extraites en pratiquant l'égouttage à la machine.

$$
\text { C. - Contrôles }
$$

Toutes les lactations ont duré au moins 300 jours pendant lesquels les deux lots ont subi les contrôles suivants :

I. Production laitière.

Le lait de chaque vache a été pesé chaque jour, matin et soir, pendant toute la durée de l'expérience.

2. Composition du lait.

Les dosages ont commencé en moyenne 25 jours après la mise bas et se sont répétés à chaque traite au rythme de :

a) Une semaine sur 4 pour l'appréciation du taux butyreux (méthode de Gerber) ;

b) Une semaine sur 8 pour la mesure:

-- de la teneur en matières protéiques au noir Amido,

- de la teneur en lactose par la technique de Somogyi (I952). Ce contrôle a été remplacé en seconde année par l'évaluation des matières sèches totales après dessiccation au bain-marie puis à l'étuve à vide.

\section{Irritation et état sanitaire de la mamelle.}

La numération sommaire des leucocytes du lait de chaque quartier a été réalisée une fois par mois sur tous les animaux, par le California Mastitis test (CMT au teepol bromocrésol pourpre).

Cette numération a toujours eu lieu 6 jours après la traite omise, afin que le contrôle ne soit pas faussé par l'effet passager et légèrement traumatisant qui résulte de l'accumulation du lait dans la glande.

\section{D. - Expression des résultats}

Nous avons calculé la production hebdomadaire des différents constituants, ainsi qu'une moyenne quotidienne pour la semaine considérée.

Compte tenu de 1'écart séparant les différents contrôles successifs, leur production globale en 300 jours a pu être estimée à l'aide d'une méthode semblable à celle proposée par Firischman.

Les résultats du lot expérimental ont été ainsi comparés à ceux du lot témoin, mais également à ceux d'un lot intitulé " experimental corrigé ". Ce lot est obtenu en recalculant chaque semaine et pour chaque vache un rendement hebdomadaire théorique sur la base des productions des mercredi, jeudi, vendredi et samedi consilérés comme redevenus des jours normatux. Cette technique d'étude à court terme 
ne tient malheureusement pas compte des effets respectifs de la suppression hebdomadaire, qui doivent défavorablement interférer à long terme sur le potentiel sécrétoire des cellules mammaires.

\section{III. - RÉSULTATS}

\section{A. - Effets sur la production en 300 jours}

Les courbes de lactation (moyenne des 2 années) sont présentées à la figure $I$. 'n remarquera qu'au départ les lots sont bien équilibrés (1). L'écart entre les deux

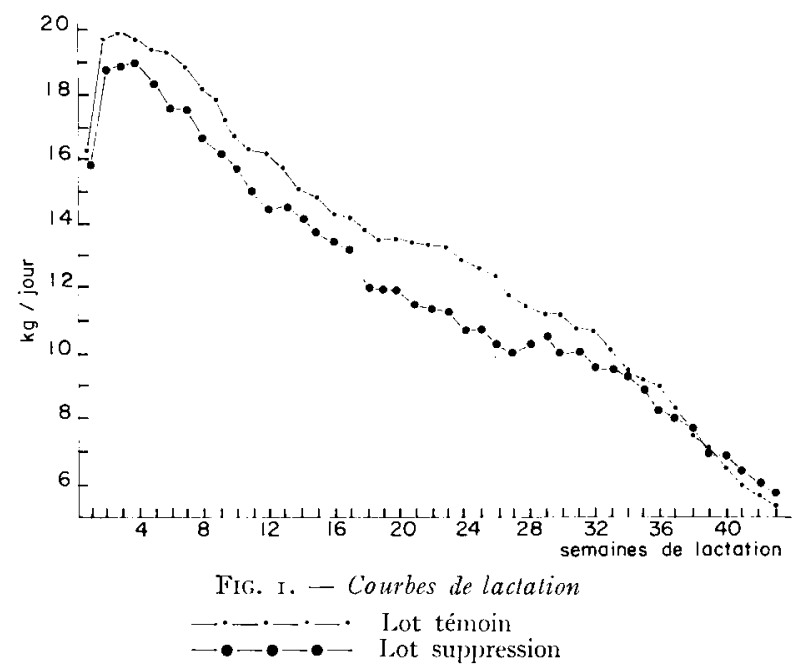

lots s'accroît légèrement pendant les 200 premiers jours. Au-delà de cette limite, l'interprétation est rendue plus délicate, deux animaux du lot témoin ayant été malades pendant cette période (corps étranger dans le rumen, intoxication).

Les résultats concernant le rendement en 300 jours sont rapportés au tableau 2.

TABI,EAU 2

Production moyenne par vache (en $300 \mathrm{j}$ )

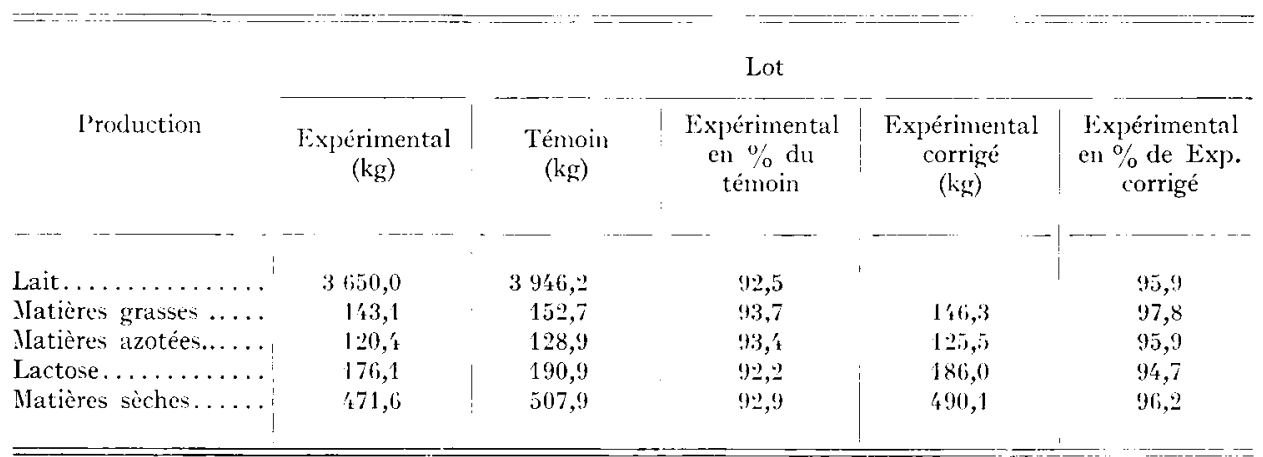

(1) Les animaux témoins produisent un peu plus que ceux du groupe expérimental, ce qui est normal puisque sur ces derniers on pratique déjà une traite en moins. 
Lorsque l'on supprime la traite du dimanche soir, les vaches fournissent seulement 92,5 p. Ioo du lait produit par celles qui sont soumises au rythme normal de traite. Cette réduction de $7,5 \mathrm{p}$. Ioo est un peu supérieure à ce qui est réellement laissé dans la mamelle, puisqu'une traite moyenne du soir représente environ 6 p. Ioo du total hebdomadaire.

La perte la plus faible est celle qui intéresse les matières grasses (6,3 p. I00), la plus forte est celle du lactose $(7,9 \mathrm{p}$. I00). Ces pertes sont nettement inférieures lorsqu'on fait porter la comparaison sur le lot expérimental corrigé. Nous avons vu au chapitre précédent ce que nous devions penser de cette seconde méthode.

\section{B. - Modifications journalières de la production et de la composition du lait}

\section{Production laitière.}

Le lait du lundi matin est abondant: I0,86 kg au lieu de $7,42 \mathrm{~kg}$ habituellement (fig. $2 a$ ).

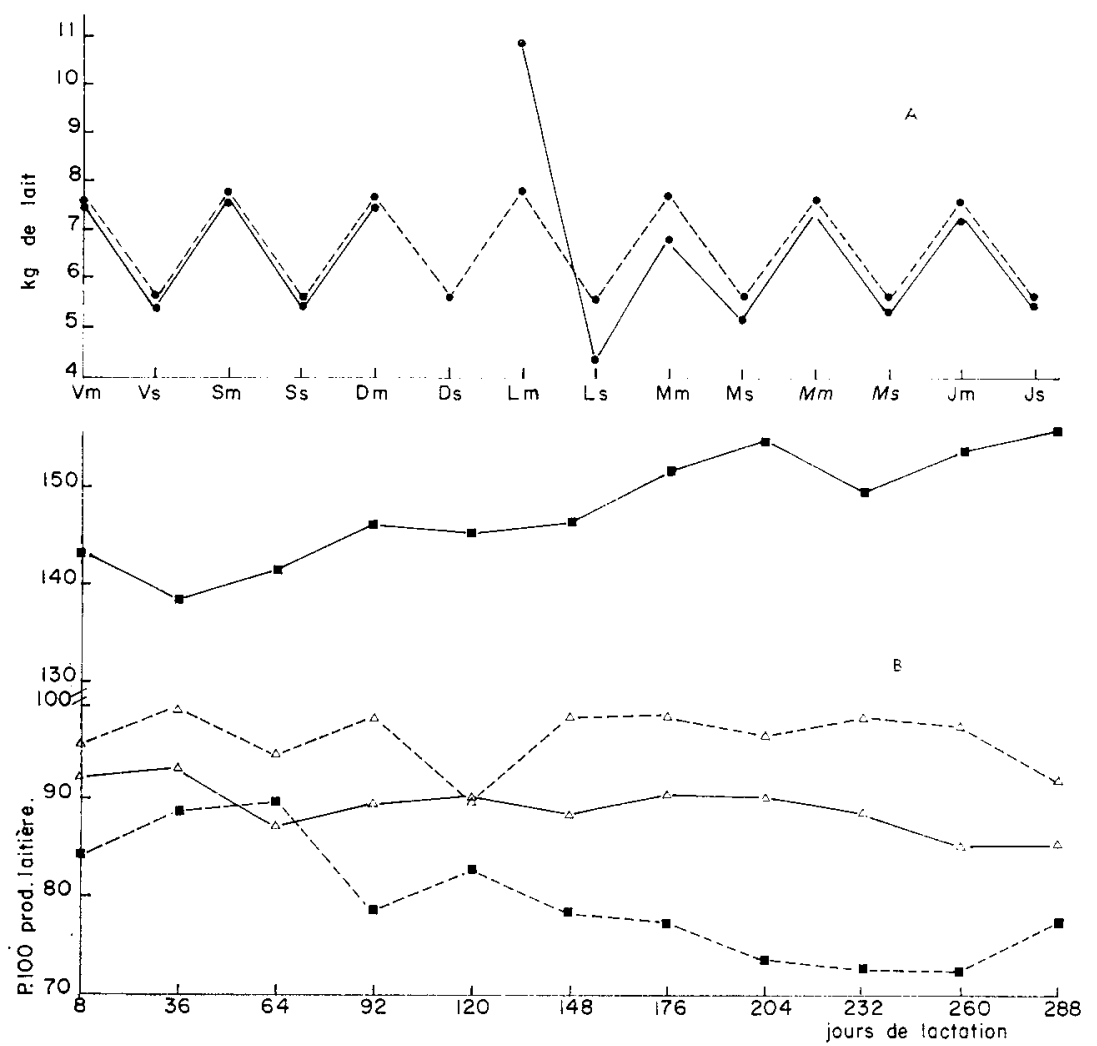

FIg. 2. - Production laitière

a) Modifications journalières (moyenne de tous les contrôles)

b) Évolution relative du lundi matin du lundi soir $\cdots$ mardi matin $-\Delta \ldots \Delta \ldots \Delta \ldots$
du portées en p. roo du mardi soir ... $\Delta_{\ldots} \Delta_{\ldots} \ldots \Delta_{\ldots}$ d'une traite normale 
Bien que permettant de recueillir une bonne partie de ce qui avait été laissé dans la mamelle le dimanche soir, ceci représente une perte de $r, 83 \mathrm{~kg}$ par rapport aux quantités totales obtenues lorsque l'on pratique deux traites par jour (12,69 kg).

La sécrétion ayant été probablement normale pendant toute la journée du dimanche, cette perte est certainement imputable à l'intervalle nocturne du dinanche au lundi, pendant lequel il n'a donc été produit que : 7,42 kg-I,83 kg = 5,69 kg. La sécrétion pendant cet intervalle a donc été freinée de $23,4 \mathrm{p}$. Ioo.

Le lundi soir et le mardi matin, la production est également sensiblement réduite, mais de moins en moins (respectivement $4,28 \mathrm{~kg}$ au liet de $5,27 \mathrm{~kg}$, soit $\mathrm{I}_{7}, 8 \mathrm{p}$. IoO et $6,68 \mathrm{~kg}$ au lieu de $7,42 \mathrm{~kg}$, soit Io p. IOo).

Toutes ces données qui sont celles d'une " semaine moyenne type " évoluent au cours de la lactation. Nous les avons rapportées en pourcentage d'une traite normale correspondante, dans la figure $2 b$.

Ainsi, la production relative du lundi matin augmente du $3^{\mathrm{e}}$ jour ( $13^{8}, 3 \mathrm{p}$. Ioo) au $288^{\mathrm{e}}$ jour ( $555,74 \mathrm{p}$. IOo) alors qu'inversement celle du lundi soir diminue de $90 \mathrm{p}$. IoO à $72,6 \mathrm{p}$. I00. Ces deux phénomènes se compensent peut-être et leur interprétation physiologique sera discutée ultérieurement. Le mardi soir, la courbe évolu-
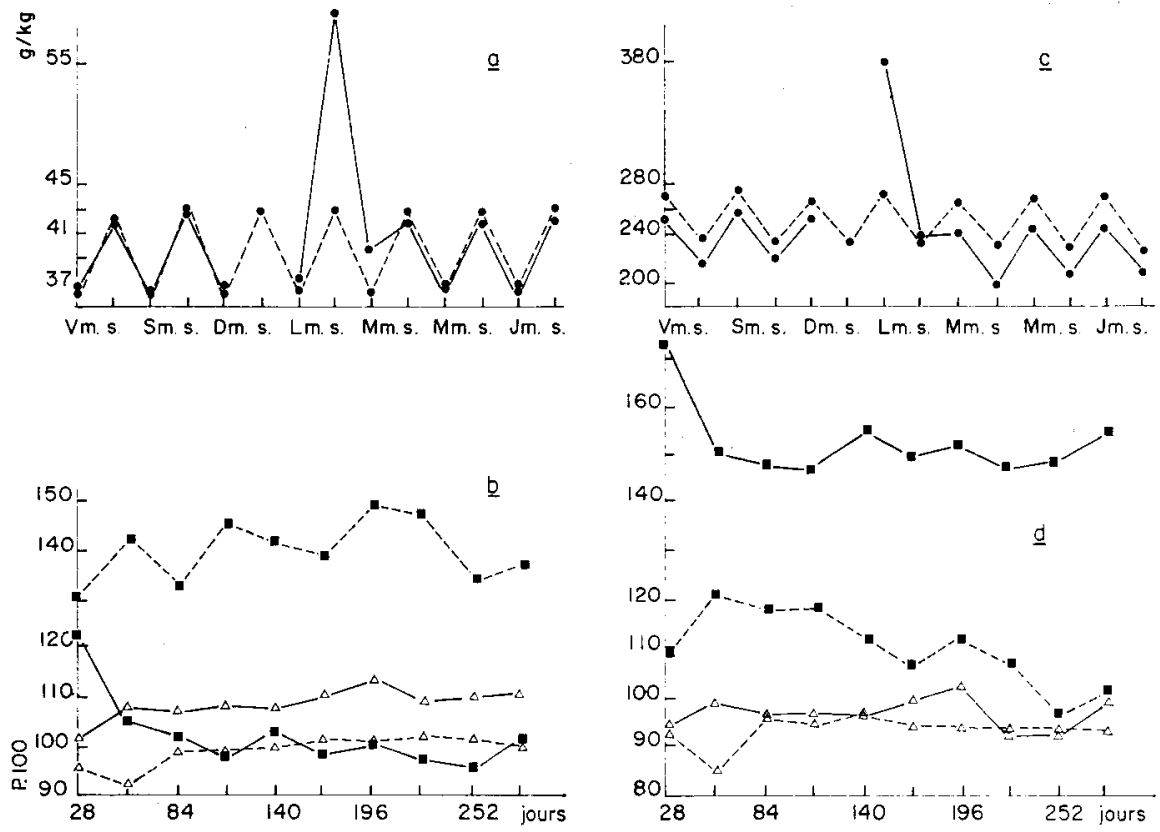

140

$\underline{\text { d }}$

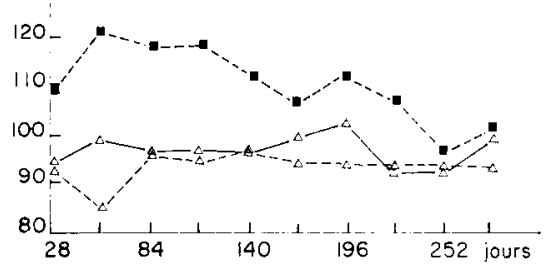

FIG. 3. - Matières grasses

Modifications journalic̀es moyennes

a) du taux butyreux

c) des matières grasses totales

Evolution au cours de la lactation

b) du taux butyreux (en p. 100 d'une traite normale)

d) des matières grasses totales (en p. 100 d'une traite normale)

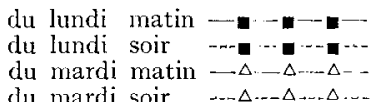

du mardi soir $\ldots \Delta . . \Delta . . . \Delta \ldots$ 
tive se situe entre $95 \mathrm{p}$. Ioo et roo p. Ioo, et on constate donc encore un léger effet dépressif.

\section{Taux butyrenx.}

Le taux butyreux moyen du lundi matin $(37,2$ p. I ooo) semble légèrement supérieur à ce qu'il est un matin ordinaire (36,4 p. I ooo) (fig. $3 a$ ). Cette supériorité est surtout due aux valeurs relevées pendant les deux premiers mois qui suivent la mise bas. Enstrite elle s'estompe et devient pratiquement inexistante (fig. $3 b$ ). Le lundi soir, le lait est beaucoup plus riche en graisse que d'habitude (en moyenne 59 p. I ooo contre $42 \mathrm{p}$. I ooo), cette perturbation augmentant très légèrement au cours de la lactation. Certains animaux peuvent même atteindre, à cette occasion, des concentrations supérieures à 85 p. I ooo. Le mardi matin, le taux butyreux est encore élevé $(39,7$ p. I ooo au lieu de 36,4 p. I ooo) et ce n'est que le mardi soir qu'il redevient à peu près normal.

\section{Matières grasses totales.}

Le lundi matin, compte tenu des remarques que nous venons de faire pour le taux butyreux et la production laitière, il est logique que nous obtenions beaucoup de matières grasses (fig. $3 c$ ). Celles-ci, après le premier mois de lactation, représentent environ et d'une façon stable, I5o p. Ioo de ce que l'on extrait normalement le matin (fig. $3 d$ ). Le lundi soir, la quantité de graisses recueillies est encore un peu forte (236,3 g au lieu de 2 I I,5 $\mathrm{g}$ en moyenne). Fille devient au contraire un peu faible aux deux traites du mardi, qui sont elles aussi perturbées.

\section{Taltx azoté.}

On constate une légère augmentation du taux azoté le lundi matin (33, I p. I ooo au lieu de 32, I p. I ooo) et une augmentation plus forte le lundi soir $(35,9 \mathrm{p}$. I ooo au lieu de 34 , I p. I ooo) qui se traduit exceptionnellement chez certains animaux par des concentrations avoisinant $45 \mathrm{p}$. I ooo, surtout à l'approche du tarissement (fig. $4 a$ et $4 b$ ).

La suppression de la traite du dimanche soir modifie done le taux azoté dans le même sens que le taux butyreux, mais à un degré beaucoup moindre. Le mardi matin en particulier, la composition du lait pour ce constituant est redevenue à peu près normale et représentative de la valeur de l'animal. Si l'on sait qu'au cours de la traite la teneur en matières azotées diffère peu entre les premières et dernières fractions recueillies, on comprend mieux que les facteurs du milieu, pouvant provoquer des rétentions de lait (frayeurs, grands intervalles de traite) soient finalement moins ressentis.

Tous ces arguments sont en faveur de la prise en considération du taux azoté lors des opérations de contrôle laitier. Il serait bon d'en tenir compte là où la suppression de la traite du dimanche soir serait adoptée.

\section{Matières azotées totales.}

Comme pour les matières grasses, la quantité de matières azotées est très forte le lundi matin : $355,3 \mathrm{~g}$ contre $236,2 \mathrm{~g}$ ordinairement (fig. $4 \mathrm{c}$ ), soit environ $5^{\circ} \mathrm{p}$. IOO 
( I $_{42,2}$ p. Ioo le $28^{\mathrm{e}}$ jour avec un accroissement régulier jusqu'à I55,76 p. Ioo le $25^{2}$ jour) (fig. $4 d$ ).
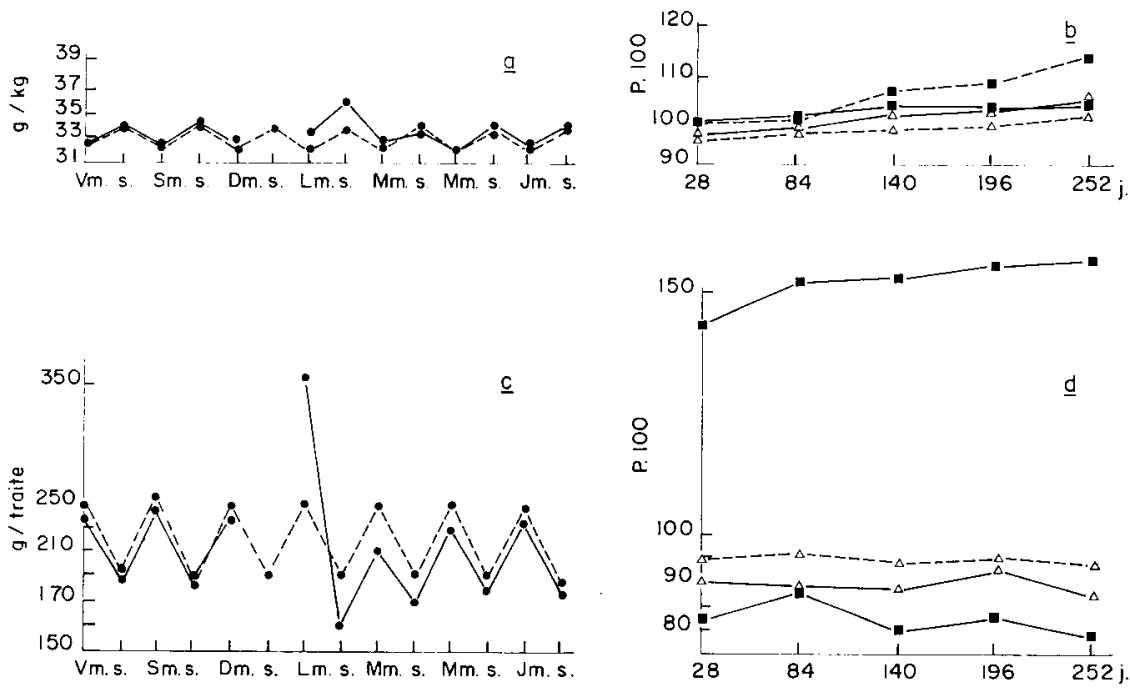

FIG. 4. - Matières azotées

Modifications journalières moyennes

a) du taux azoté

c) des matières azotées totales

Evolution au cours de la lactation

b) du taux azoté (en p. Ioo d'une traite normale)

d) des matières azotées totales (en p. Ioo d'une traite normale)

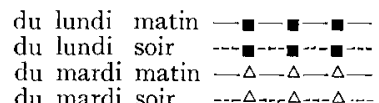

Mais cette fois, la production du lundi soir est plus faible que d'habitude (I49, I g au lieu de $\mathrm{I} 79, \mathrm{I} g$ ). C'est également vrai le mardi matin et le mardi soir et dans ces trois derniers cas le phénomène change peu au cours de la lactation.

\section{Taux de lactose.}

Les résultats diffèrent de ceux obtenus pour les taux butyreux et azotés. En effet, il n'existe pas de " compensation " consécutive à la suppression de la traite, mais au contraire un appauvrissement en sucres sur trois traites au moins (fig. $5 a$ ) :

48,78 p. I ooo le lundi matin au lieu de 49,4 p. I ooo
48,92 p. I ooo le lundi soir -5 50,54 I ooo
48, I 6 p. I ooo le mardi matin $-\quad 49,4$ p. I ooo

Ces modifications sont toutefois très faibles et restent pratiquement identiques d'un mois à l'autre (fig. 5 b).

\section{Lactose total.}

Malgré les faibles concentrations en lactose enregistrées le lundi matin, la production globale de ce constituant représente tout de même $130,53 \mathrm{p}$. Ioo de celle d'une 
traite normale au premier contrôle, et $\mathrm{I43,4 \textrm {I }}$ p. IOO au dernier (fig. $5 c$ et $5 d$ ). Enn revanche, la réduction est sensible le lundi soir et ceci d'une façon d'autant plus intense qu'on s'éloigne de la mise bas.

Cette remarque est également valable pour les deux traites suivantes (fig. $5 d$ ).
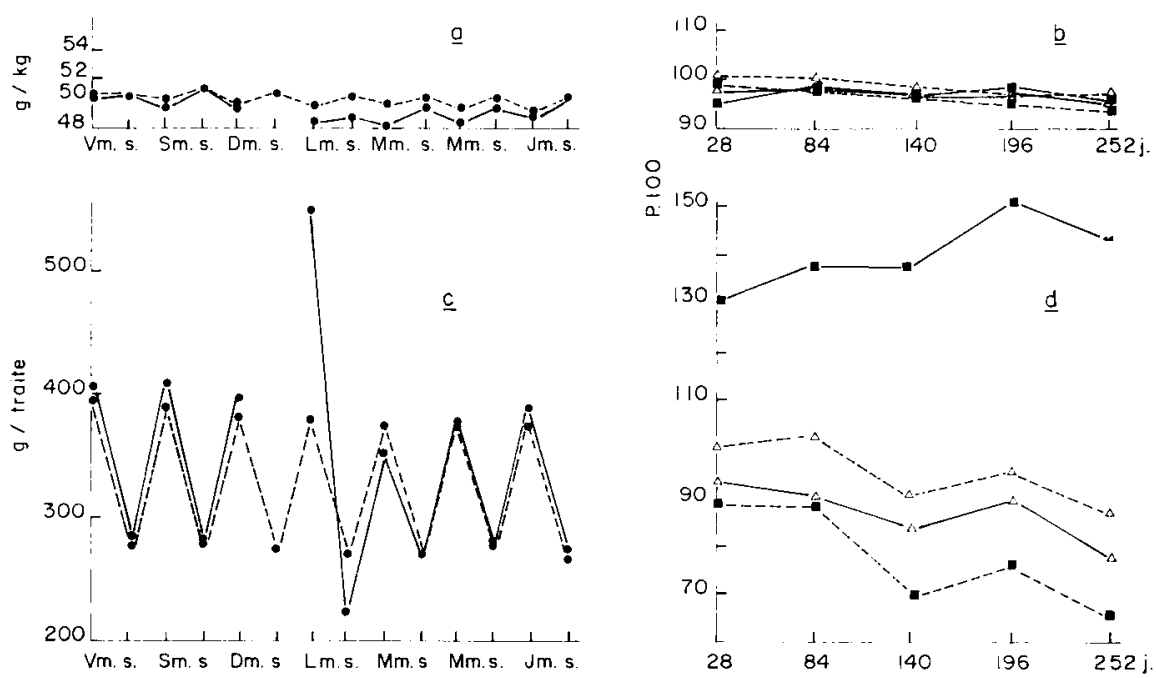

Fig. 5. - Laclose

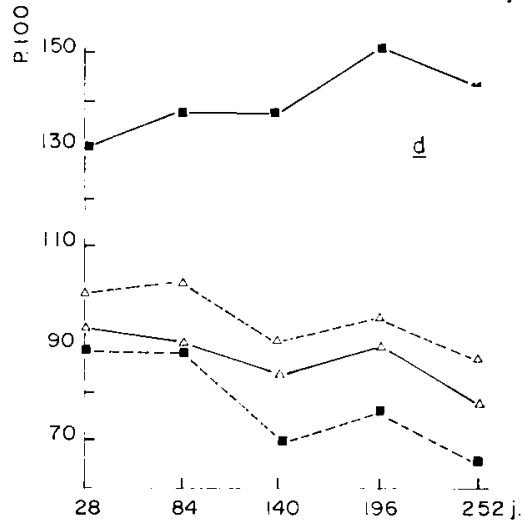

Modifications iournalières

a) du taux de lactose

c) du lactose total

Evolution au cours de la laciation

b) du taux de lactose (en p. soo d'une traite nomale

l) du lactose total (en p. roo d'une traite normale)

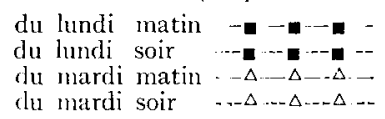

8. Taux de matières sèches.

La teneur en matières sèches (fig. 6 a) est la résultante des modifications journalières de la concentration en azote, lactose et surtout en matières grasses, c'est-àdire :

- teneur pratiquement normale le lundi matin : I22, I6 g p. I ooo au lieu de 123.27 p. I ooo;

- beaucoup plus forte que d'habitude le lundi soir : I5I,78 p. I 000 au lieu de I3I,o p. I 000 ;

- encore élevée le mardi matin : r26,6 p. I 000 au lieu de I23,27 p. I 000.

L'ampleur relative de ces modifications n'est pratiquement pas affectée par le stade de lactation (fig. 6 b).

9. Extrait sec total.

Très forte le lundi matin (I.272,6 g), la production totale de matières sèches semble ensuite systématiquement inférieure à ce qu'elle devrait être, au moins jusqu'au jeudi (fig. 6 c). Ce critère, qui schématise assez bien l'intensité de fonctionne- 
ment de l'épithélium mammaire, nous indique donc que les phénomènes sécrétoires sont encore légèrement modifiés au-delà des 48 heures qui suivent l'omission de la traite.

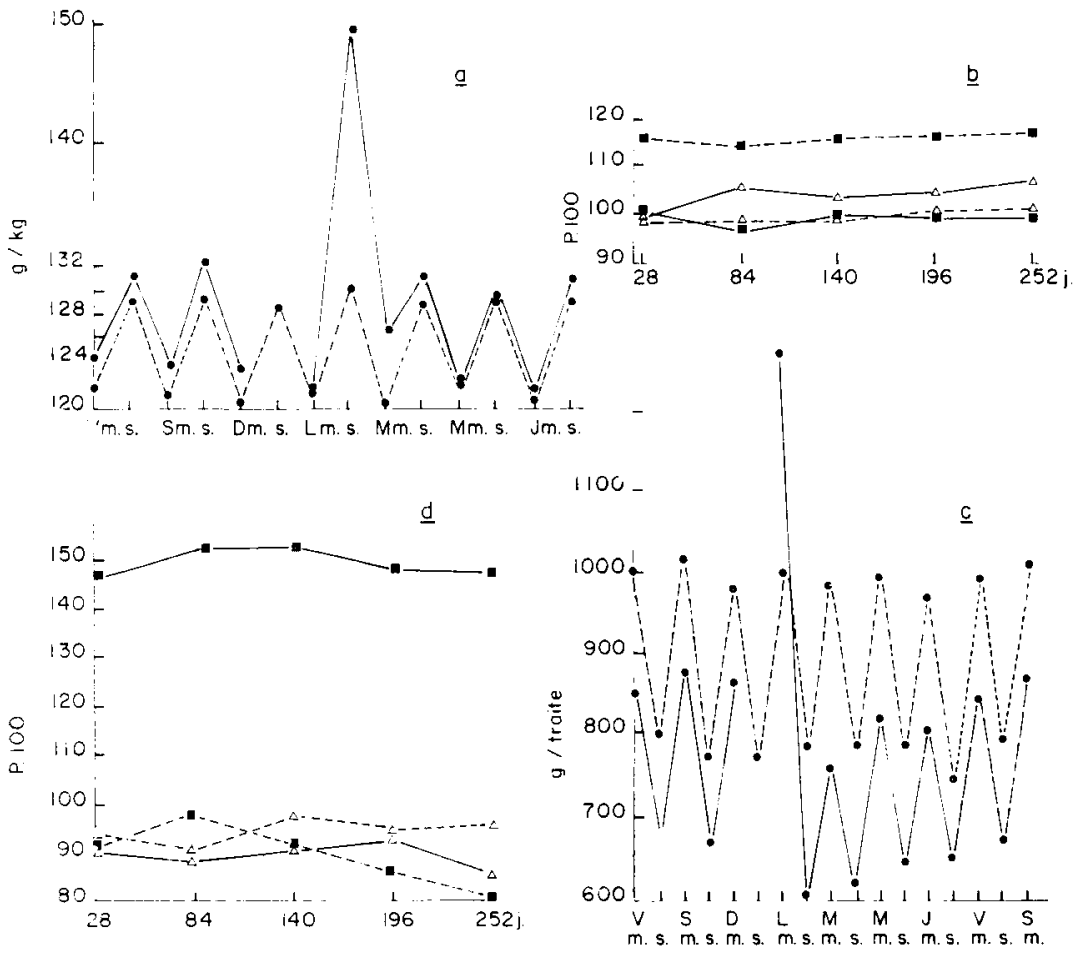

Fifg. 6. - Malières sèches

.Mollifications journalières movennes

a) du taux de matières sèchès

c) des matières sèches totales

lizolution alt cours de la lactation

b) du taux de matières séches (en p. 100 d'une traite normale)

d) des matières sèches totales (en p. 100 d'une traite normale)

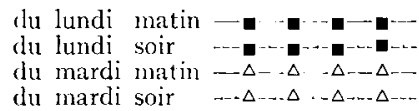

Un examen attentif de la figure $6 c$ semblerait même prouver que de tels effets, si faibles soient-ils, pourraient se prolonger jusqu'au dimanche suivant. La nouvelle suppression interviendrait alors avant même que le métabolisme ne soit redevenu normal.

\section{L Itat sanitaire de la mamelle}

Pour le lot expérimental, le nombre de cellules dans le lait reste en moyenne toujours inférieur à 500 o0o $/ \mathrm{ml}$, c'est-à-dire en dessous des limites considérées comme indiquant une mammite subclinique. 
L'évolution de la notation $C M T$ est identique dans les deux groupes d'animaux, les vaches témoins réagissant d'ailleurs d'une façon plus sensible au test au teepol (fig. 7).

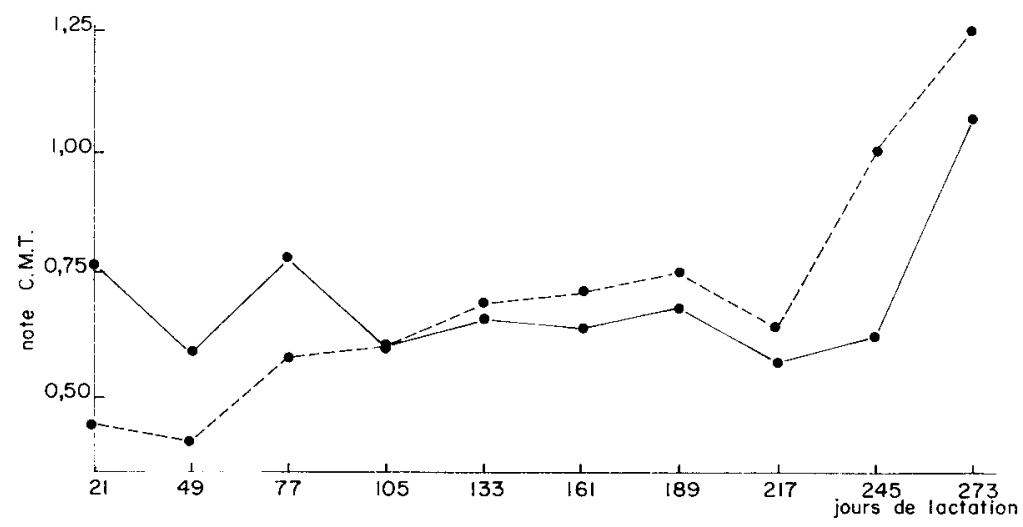

FIG. 7. - Évaluation sommaire du nombre de cellules dans le lait au cours de la laciation (test CMT)

$$
\begin{aligned}
& \text { Lot suppression } \\
& \text { Lot témoin } \\
& 0,50 \text { environ } 2 \times 10^{5} \text { cellules } / \mathrm{ml}
\end{aligned}
$$$$
\text { I, }, 0 \text { environ } 4 \times 10^{5} \text { cellules } / \mathrm{ml}
$$

L'augmentation du nombre de cellules constatée à l'approche du tarissement est un phénomène normal à ce stade de la lactation.

En outre, il est intéressant de noter que certaines vaches, subissant la suppression de la traite, et présentant dès la mise bas des réactions positives sur certains quartiers (reste d'infections bactériologiquement guéries mais non cicatrisées), n'ont jamais évolué vers une mammite du type aigu.

\section{IV. - DISCUSSION}

Quatre points principaux se dégagent des nombreuses observations que nous venons de formuler.

I. La suppression de la traite du dimanche soir entraîne, sur 300 jours, une réduction de la production des matières grasses, des matières azotées, du lactose, de l'extrait sec et du lait, respectivement de $: 6,3$ p. Iоo; 6,6 p. Iо0 ; 7,8 p. Ioo ; 7, I p. IOO; et 7,5 p. IOO.

Ces valeurs, qui sont du même ordre que celles enregistrées par CIAEsson, ne confirment que partiellement les travaux de AUTREX, ROLLINS et CANNON (I963) puisque, contrairement à ces auteurs, nous constatons une diminution non négligeable de la quantité totale de matières grasses.

Enfin, et bien que travaillant dans des conditions expérimentales différentes (expérimentations chez l'exploitant), DARRaco ( $\left.{ }^{96} 67\right)$ aboutit à des résultats qui, pour l'essentiel, viennent corroborer nos propres données. 
Il serait intéressant de vérifier si la perte de production occasionnée par la suppression de la traite du dimanche soir, entraîne, comme on peut le supposer, une diminution appréciable des besoins alimentaires chez les animaux soumis à ce traitement.

Compte tenu également des économies de main-d'œuvre qu'il est aisé de chiffrer, chaque éleveur serait alors en mesure de faire un bilan financier adapté aux conditions d'exploitation de son troupeau.

2. La production et la composition du lait sont fortement perturbées pendant les 48 heures qui suivent 1'omission de la traite. Pratiquement, ceci interdit le passage du contrôleur laitier avant le mardi soir, dans les fermes où cette technique serait adoptée.

Comme le proposent WHEELock, RoOK et DODD (I965), il est possible que pendant les 24 heures d'accumulation du lait dans la glande, l'accroissement de la pression intramammaire modifie le flot sanguin et la perméabilité des membranes de l'épithélium sécrétoire.

Ainsi, des phénomènes de réabsorption d'ezu et de lactose (WHEELock et Rook, I966) pourraient expliquer la faible teneur en sucres du lundi matin. Ils devraient inversement conduire à une concentration importante des matières grasses et des protéines. Or, ceci ne se produit qu'à la traite du lundi soir et il faudrait donc admettre pour expliquer les teneurs normales du lundi matin, que:

a) les protéines soient également réabsorbées comme l'ont suggéré VERIEY et HOLimanN (Ig66).

b) les taux butyreux élevés soient masqués par une forte rétention des graisses dans les parties supérieures de la glande. On sait en effet, que la lait résiduel est d'au. tant plus important que l'intervalle qui précède la traite a été grand.

Toutefois, ces considérations sont particulièrement hasardeuses, et en réalité ne sont justifiées par aucun argument expérimental décisif.

De plus, on comprend difficilement les modifications de la composition du lait constatées le lundi soir, le mardi et même le mercredi et le jeudi. Cela ne peut être dî encore à des phénomènes de réabsorption sélective, puisque 4 heures après le rétablissement de la traite, WHEELOCK et ROOK (Ig66) retrouvent une lactosémie et une lactosurie négligeables et donc une perméabilité membranaire normale. Il faut donc supposer que d'autres mécanismes plus complexes encore entrent en jeu, et qu'en particulier l'activité sécrétoire des cellules a été fortement perturbée.

Ce ne sont là que des hypothèses, et une étude approfondie mériterait d'être entreprise sur les causes des modifications de la composition du lait, consécutives à des grands intervalles de traite.

3. Rapportée à la production d'une traite normale, la quantité de lait relative du lundi soir augmente avec le stade de lactation. Ce phénoméne signifie peut-être que l'activité synthétisante des cellules diminue plus vite que les capacités de stockage de la mamelle. Ainsi, disposerait-on de plus en plus de temps avant que le volume creux de la glande ne soit rempli et la sécrétion ralentie.

4. Comme 1'avait déjà mentionné ClaEsson (I965), l'état sanitaire du pis ne semble pas s'aggraver lorsque l'on supprime une traite par semaine. Il convient toutefois de rester prudent en ce domaine, la prédisposition aux mammites étant souvent un facteur d'étable. 


\title{
REMERCIEMENTS
}

Nous tenons à remercier MAI. E. L.egrndre, A. Robin et J. l'Esigot, pour l'aide précieuse qu'ils nous ont apportée lors de la réalisation de ce travail.

\section{SUMMARY}

\author{
EFFECT OF OMITTING ONE MILKING ON SUNDAY \\ IN " FRENCH FRIESIAN PIE NOIRE " COWS
}

The evening milking was omitted on Sunday for the duration of one lactation cycle in 14 French Friesian lie Noire cows (table $\mathrm{r}$ ). There was no alteration in the times of other milkings.

In comparison with a control batch, the total milk yield in 300 days was 7.5 per cent lower. The fat, nitrogen, lactose contents and dry matter were $6.3 ; 6.6 ; 7.8 ; 7.1$ per cent lower respectively (table 2 ).

The yield and composition of milk have been considerably disturbed for the first 48 hours following the omission of milking (graphs 2, 3, 4, 5, 6). (on Monday mornings, fat and nitrogen contents were slightly higher : lactuse rate on the contrary was lower. On Monday evenings, fat and nitrogen contents were very high, while lactose rate was still depressed.

The original composition was recovered within the next two milkings and the milk inspector's visit should not take place before Tuesday evening.

A thorough examination of the evolution of dry matter (graph $6 c$ ) would incline us to the view that the recovery period could last until the next sunday.

No alteration in udder health was noticed, even after a long period (graph 7 ).

\section{RÉFÉREITCES BIBLIOGRAPHIQUES}

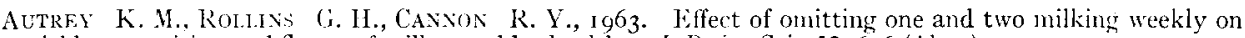
vield composition and flavor of milk on uddar health. J. Dairy $S c i ., 46,626$ (Abst.).

$130 G \mathrm{NeR}, 1063$. Effect of leaving out a milking on the weekly yield of cows during pasture and stall maintenance. Titrathter, 15, 627-620).

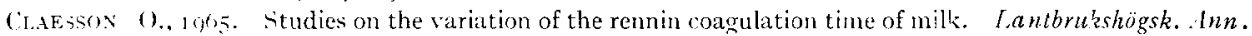
$31,237-3,32$.

Ctafssox O., Inxison A. Gustafsson X., Brannani E., r959. Situdies on monozygous cattle twins. XVIT. Once-a-day milking compared with twice-a-day milling. Act. agr. Scand., 9, $3^{8-58 .}$

DiRrac! J.. Int). Communication personnelle.

ELLIOT (i. 11., Io5y. The direct effect of milk accumulation in the udd er of the dairy cow upon milk secretion rate. Laim Sci. Ibstr., 21, $+35^{-}+39$.

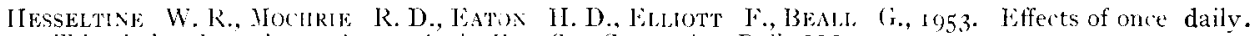
milking in latte latation. Siorrs. Agric. Exp. Sta. Connecticul Butl., 304, 10.

I.ABCsitike I.. RICHARI) T'h., 1965. La traite mécanique. Aspects anatomiques, physiologiques et terhnologiques. lîine atu point bibliographique. Ann. Zo tech. 14, 63-126.

Sosiony M., 1952. Notes on sugar determination. J. Biol. Chem., 195, ic).

Vhrley J. H., Holluivin K. II., sg60. Synthèse et réabsorption des protéines dans la glande mammaire en stase. Fiude antoradiographique au nicroscope électronique. Z. Zellforsch, 75, 605-6io.

WheELock J. V., Rook J. A. F., i 966 . Lactose in the blood and urine of cows. J. Dairn. Res., 33, 37-42.

Wirfelock J. Y., ROOK J. A. F., DOL], F. H., 1965. The effect of incomplete milking or of an extended milking interval on the yield and composition of cow's milk. J. l)airy Res., 32, $237^{-2}+7$.

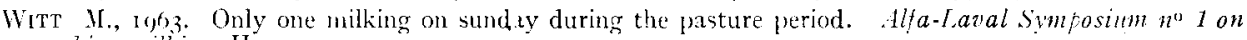
machine milking, Hamra, 200-203.

WOODWARI' T'. H., I931. The preduction of dairy cows as affected by frequency and regularity of milking and feeding. l. S. Dept. Igr. Cir., 180, a-16. 